\title{
RESEARCH AND DEVELOPMENT IN HAEMATOLOGY. A REPORT ON INTERNATIONAL CONGRESSES AND VISIT TO ACADEMIC CENTRES IN EUROPE
}

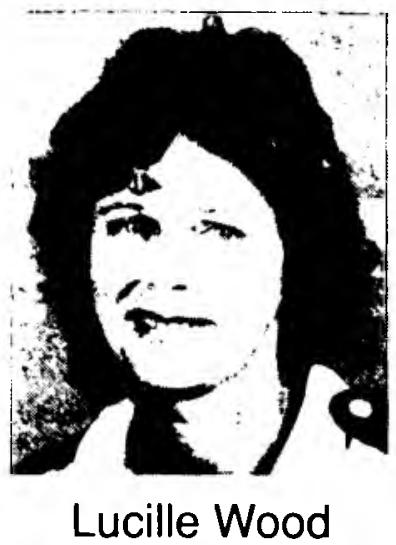

Transplantation Group in Badgastein, Austria. A copy is available for interested people.

departmental research at the Intermational Bone Marrow Transplantation Meetings and to visit a number of academic centres overseas. This report summarises those experiences, with particular emphasis on the role of the professional nurse in research and development as it applies to contemporary haematology.

\section{OBJECTIVES}

1. To accept an invitation from Professor Anton Hagenbeek at the Daniel den Hoed Cancer Centre in Rotterdam, Holland, to see in operation the bone marrow transplantation programme, with an indepth review of their antibiotic policy that includes selective decontamination of the bowel.

2. To present a paper at the Second International Symposium on Acute Leukaemias in Munster, Federal Republic of Germany, detailing experiences from the Department at the University of Cape Town in treating adult acute lymphoblastic leukaemia. A copy of the manuscript is available for interested people.

3. To visit Professor Wolfgang Hiddeman and attend ward rounds, with discussions on the management of haematologic malignancy as well as bone marrow transplantation at University Hospital in Munster, Federal Republic of Germany.

4. To accept an invitation from Professor Bruno Speck at the Kanton Hospital in Basle, Switzerland, for discussions, particularly the care of immunocompromised patients in protected environments.

5. To present a paper at the XVth Annual Meeting of the European Bone Marrow
6. To attend the Vth Meeting of the European Bone Marrow Transplantation Nurses Group in Badgastein, Austria.

7. To accept an invitation from Professor Paul Imbach to attend research meetings in Basle and Bem to discuss our interim results on the management of patients with immune thrombocytopenia using highdose intravenous gammaglobulin. A copy is available for interested people.

8. To review at each of these centres current practice and future direction for the optimum use of continuous-flow blood fraction separators in support programmes for bone marrow transplantation.

9. To compare nursing practice and problems at each institution with those that we have developed in our department at the University of Cape Town.

\section{REPORT ORGANIZATION}

Because of inevitable duplication of information from the various meetings and visits to the university hospitals and academic haematologic advances by disease category, followed by data on operation of the protected environment for management of immunocompromised patients, the use of continuous-flow separator technology and, finally, bone marrow transplantation.

\section{DISEASE CATEGORIES}

Cell Biology and Acute Leukaemla These topics were a prominent part of all meetings and discussions.

Cytogenetic studies are now routinely being explored to document the occurrence of centres, this is organised to deal, firstly, with non-random changes in preleukaemia, de novo, and secondary leukaemia. Furthermore, specific structural changes, usually translocations, are being correlated with particular leukaemic variants. However, where karyotypic changes occur infrequently, any significant association with one or other morphologic subtype awaits finality.

Examples for both diagnosis and estimation of prognosis include $t(15 ; 17)$ in preleukaemia where there is a low incidence of transformation, $t(6 ; 9)$ in acute nonlymphoblastic leukaemia, and both $t(9: 22)$ and $t(4 ; 11)$ in acute lymphoblastic leukaemia predicting for poor response to therapy and short duration of survival. Such studies are useful, when carried out serially, to recogr early relapse after either chemotherapy or bone marrow grafting.

Another topic is the increasing use of monoclonal antibodies to deplete the Tlymphocyte populations thought to mediate graft-versus-host disease (GvHD) following bone marrow transplantation, but such manipulations may be associated with an increased risk of leukaemic relapse. Studies from Israel show that, at least following autgrafting, ex vivo activation of residual mononuclear cells with a mixture of interferon and interleukin 2 appears to have an antileukaemic effect by reducing relapse rates. In further studies the gradual reintroduction of donor peripheral T-cells, obtained by continuous-flow separator technology, seems to have a similar effect. In both these situations GvHD did not occur. despite neither cyclosporin nor methylprednislone being used for immunosuppression in the post-transplant patient.

In the context of our own practice it is clearly necessary to characterise all patients morphologically, immunophenotypically, and cytogenetically at presentation - the concept 
of the MIC classification. Our commitment to use this nomenclature for analysis of remission, survival and relapse is consistent with international practice.

It was disappointing to find that no reference was made to the studies reported from our group, jointly with Professor Lyn Wilson, on plasminogen activator status; it remains uncertain as to whether anybody else is repeating this work.

\section{Acute Nonlymphoblastic Leukaemia \\ (ANLL) in Adults}

Predictably, these studies were remarkable for the diversity of chemotherapy programmes employed throughout the world. There is a recognisable trend in which high-dose cytosine arabinoside, often in combination with Amsacrine or $\mathrm{m}$-AMSA, is being used as consolidation or during maintenance; increasing toxicity is evident in the older age groups.

On a worldwide basis there seems to be consensus that an anthracycline, in combination with conventional-dose cytosine inoside, are the agents of choice, although immulticentre studies even these tended to give results somewhat lower than the remission rates of $70 \%$ being claimed in some centres. Primary drug resistance remains a major problem and occurs in 7 to $15 \%$ of patients. One of the contentious issues in all these studies is definition of complete remission. Generally speaking, marrow examination at day 14 might be free of morphologically recognisable leukaemia, but this does not guarantee remission. Of note was the converse observation that existence of myeloblasts at this time of study does not mean that the patient will fail to achieve remission. Not surprisingly, therefore, centres tend to have their own roughly similar practice of evaluating the adequacy of their chemo-therapy programmes.

One of the striking problems is that of the continuing and random relapse that follows achievement of remission in response to any of the currently accepted first-line chemopy programmes. To try and overcome this risk is emerging a consensus in favour of bone marrow transplantation. The procedure might either be allogeneic, autologous or, as we use in our department, doubleautografting. In all these situations recombinant growth factors, particularly in the form of granulocyte colony stimulating factor, remain a promising avenue for research.

\section{Acute Nonlymphoblastic Leukaemia (ANLL) in Children}

Again, treatment programmes varied widely, with strong support for the entry of patients using stratification based on prognostic factors at presentation. Specifically, age and elevated white cell count predict for poor outcome following induction chemotherapy. Surprisingly, and only in children, a marrow aspiration carried out at day 14 following commencement of initial treatment appears to have predictive value for remission. As in adults, high-dose cytosine arabinoside is being increasingly explored, but it remains uncertain as to whether its role is most useful as part of induction as opposed to consolidation or intensification phases of treatment. The concept of less aggressive initial therapy was confirmed to be without benefit.

Of note is the increasing shift of paediatricians to the employment of bone marrow transplantation and many parallels are now emerging that have indications similar to the use of these procedures in the older age groups.

\section{Acute Lymphoblastic Leukaemia (ALL) in} Adults

A major problem remains relapse in the central nervous system, and high-dose cytosine arabinoside is being tested during early therapy on the basis that therapeutic levels of the agent are achieved in the brain and spinal cord by easy passage across the blood-brain bartier. However, in the broader experience, relapse rates in this anatomical site remain in the region of $15 \%$.

Studies using high-dose cytosine arabinoside also emphasize the significant toxicity of this regimen, with extensive skin erythema and a substantial mortality. As with the Cape Town experience, disturbed cerebral and cerebellar function occur, but to a lesser extent than encountered in patients with ANLL. The latter finding is possibly explicable on the fewer number of courses used in patients with ANLL.

Again, bone marrow transplantation is a clearly acknowledged form of treatment, particularly as consolidation in patients achieving first complete remission. It is also of value in the subgroup characterised by cytogenetic abnormalities, elevated white cell count, the presence of leukaemia in the central nervous system and, most particularly, slow response to therapy.

\section{Acute Lymphoblastic Leukaemia (ALL) in Children}

Herc, impressive data was presented from the groups in Boston and Memphis who, contrary to much of the popular opinion, emphasized the ominous significance of this disease, even in childhood. As a consequence the importance of prognostic factors in stratification of patients to more aggressive regimens is established as a fundamental approach to management.

Much effort is being devoted to reducing long-term risks associated with maintenance periods that extend beyond two years. Striking neuropsychological abnormalities are prominent, particularly in girls under 5 years of age. To try and circumvent this problem investigators are attempting to exclude cranial irradiation from patients considered to be at standard risk, but retaining this for those presenting with poor prognostic factors. In our group, albeit largely an adult series, relapse in the central nervous system has been less of a problem, possibly reflecting our early use of triple intrathecal drugs.

Furthermore, it appears as though the fully developed adult brain tolerates radiation better than that of a child and, accordingly, there currently appears less need to withdraw irradiation in individuals with a fully mature central nervous system.
There were reports of impaired growth, damaged cardiac muscle and disturbances in conduction related to chemotherapy. None of these findings have been significant occurrences in our adult patients.

High risk groups are defined as those having the presence of the Philadelphia chromosome or total white counts $>20 x$ $10^{4} / \mathrm{L}$, but when this figure exceeded $100 \mathrm{x}$ $10^{4} / \mathrm{L}$ it became an absolute indication for aggressive therapy. The rate of response to initial treatment has been identified as an indicator of poor prognosis, together with the presence of central nervous system disease and age below 2 or above 10 years.

Statistically, two-thirds of the patients at American centres fell into a high risk group.

\section{Salvage Chemotherapy}

Most centres have reached agreement that duration of remission is the best predictor for patients who, should they relapse, will achieve a second complete remission. Conversely, use of the MIC classification, with particular emphasis on chromosomal abnormalities, has been shown in a number of centres, including our own, to identify a subgroup where remission is likely to be of short duration. It is therefore important to characterise in detail both presentation features and the duration of remission, so that where a successful induction is achieved patients can promptly undergo bone marrow transplantation.

A different problem is that of primary drug resistance, and while there is debate about optimum treatment, many groups favour the use of high-dose cytosine arabinoside in combination with mitoxanthrone for ANLL. A similar situation for ALL would again favour high-dose cytosine arabinoside, but here it is combined with etoposide and methotrexate followed by leucovorin rescue. In all these situations, it is vitally important to recognise that induction and consolidation chemotherapy, irrespective of the programme is only an essential step en route to one or other form of bone marrow transplantation.

\section{The Myelodysplastic Syndromes}

These are generally difficult to treat and the use of growth factors, as we are doing in our department, is being explored. Insufficient data is presently available to make recommendations about longer term management. There is, however, clarity that in patients under 50 years of age and where a suitable donor is available, bone marrow transplantation is the treatment of choice

\section{Chronic Granulocytic Leukaemia}

The previous use of alkylating agents in the form of myeleran has been replaced by hydroxyurea. The latter is much safer and leads to more predictable recovery of myelosuppression when inadvertent overdose occurs.

The most striking changes are the way in which biologic immune response modulation, in the form of recombinant human alpha interferon, is being employed. As in our own department, patients who are not eligible for bone marrow transplantation can reasonably 
be randomised to hydroxyurea in combination with equivalent doses of this cytotoxic agent and interferon.

A much more difficult problem is that of disease acceleration or blastic transformation. In both these situations the younger patients are eligible for heroic measures that include allogeneic grafting, where a donor is available, or attempts to return the disease to the chronic stable phase, and to follow this with autografting where a suitable sibling is not available or age would otherwise make the procedure hazardous. The fact that many patients still reach this late stage of their disease is an indication of how poorly chronic granulocytic leukaemia is managed.

The undoubted advance in treatment is the use of bone marrow transplantation, described more fully in a later section of this report.

\section{Chronic Lymphocytic Leukaemia}

This remains a frustrating disease to treat. There is a misconception amount general physicians that it is benign, but nothing could be further from the truth. Initially, patients will respond to relatively innocuous treatment with chlorambucil or steroids, but gradually haematopoietic reserve is eroded and ultimately marrow failure leads to death. Since many of the patients are elderly, such an approach is a reasonable option. However, in young patients or in those responding more slowly to single agent alkylator therapy, alternative options are being examined.

Little data was presented to parallel our gradually accumulating experience in Cape Town seeking to define the relative merits of intermediate or high-dose cyclophosphamide following by consolidation with fractionated sequential hemibody radiotherapy. In most centres, as with ours, return of martow towards normal provides an ideal opportunity for harvesting and cryopreservation of stem and progenitor cells as insurance against the relentless progression of the disease. Once such a situation occurs, more intensive treatment options are needed and many of these can be given safely with the administration of growth factors in combination with autologous bone marrow transplantation. Allogeneic grafting is useful in younger patients where a donor exists. However, in both these transplant situations the availability of effective regimens for disease eradication in the patient remains the subject of intensive study.

\section{Multiple Myeloa}

Individuals with this disease are difficult to manage since most or all chemotherapeutic regimens are of limited value. Not surprisingly, attempts to alter the natural history with biological immune response modulation, using the interferons, is an avenue currently being explored. Another very promising treatment option is the use of bone marrow transplantation, and here the debate centres on the best conditioning regimen since it is not clear how most effectively to eradicate residual disease in the patient. Of note is the fact that many are over the age of 40 years and in some cases beyond 70. The latter group are better suited to autologous bone marrow transplantation and, paradoxically, the use of a graft containing morphologically recognisable plasma cells does not immediately lead to regrowth of the tumour.

One area that was little discussed, although it is beginning to attract attention, is the programme that we are developing jointly with the Department of Radiotherapy. In this approach systemic irradiation, both in drug resistant patients and as a primary form of therapy in those who do not have poor prognostic factors, exemplified by marked increase in lactic dehydrogenase or $\boldsymbol{B}$ microglobulin levels, has shown early premise.

Of particular interest in patients with myeloma is the management of their anaemia. Low haemoglobin levels are often multifactorial but, particularly in the presence of renal failure, impaired endogenous erythropoietin levels may identify patients likely to respond to parenteral administration of the recombinant human hormone. This form of therapy will diminish dependence upon red cell transfusions, with its attendant hazards.

\section{Hodgkin's Disease and Non-Hodgkin's Lymphomas}

In both these categories patients are managed to an increasing extent by the use of bone marrow transplantation. It is notable that while the younger patients may benefit from allografting, there is a major shift towards the use of autologous transplantation. Two further observations are pertinent. Firstly, the early use of marrow grafting is predicted on the basis of slow response to therapy and before widespread cytotoxic damage has occurred to marrow and other tissues. Secondly, it appears likely that harvesting of haematopoietic stem and progenitor cells from the peripheral blood may offer a practical approach, even in those with bone marrow involvement by tumour.

\section{EXPERIENCES IN DATA PRESENTATION}

Although being involved in the protocol design and all aspects of managing these patients, I was nevertheless anxious at the thought of a professional nurse having to face the inquisition likely to be directed at me by some of the world's acknowledged authorities in the field of haematologic malignancy. The ordeal turned out to be less frightening since $I$ was able to deal clearly, in fact confidently, with the very many questions that arose during the presentations. I believe that this rather modest achievement was made possible by our departmental philosophy that strongly encourages nursing staff to occupy an equal position as co- investigators, to be involved in the design, and to maintain an active part in all our programmes; as a result, we can be conversant with data accumulation and its analysis.

It was a particular pleasure to find that the group in Cape Town are well recognised and most of the investigators were willing to discuss our data without apparently experiencing any difficulty caused by the fact that I was a nurse representing the University of Cape Town Leukaemia Centre and the Department of Haematology at Groote Schuur
Hospital.

Once the ice had been broken it was easier for me to approach and discuss the work of other people. From this experience it was clear that the clinical, research and developmental activities in most of the areas in our department are known to many people at the meetings. I was also unable to find by examination of posters, listening to the presentations or in subsequent discussion with scientific, medical and nursing colleagues any discrepancy between our approach or results and work in progress at other major academic centres.

Particularly in the context of haematologic malignancy it was striking to see how allogeneic and autologous bone marrow transplantation is rapidly taking hold of routine management for patients with all of those conditions where the use of cytotoxic agents is limited by bone marrow toxicity. Since, as a department, we have more than 10 years' experience with these programmes, it is noteworthy that Professor Peter Jacobs should have had the foresight to introduce a cell support programme and establish bone marrow transplantation as one of the earl commitments at our university and hospitar. As a result there have been continuing contributions to the International and European Bone Marrow Transplantation Registries, making it possible for us to keep abreast of developments being reported to the international scientific community.

It was furthermore of interest to me, during the presentations where I was personally involved, to see and discuss directly with other individuals our own conditioning programmes and the way in which growth hormones, such as erythropoietin and recombinant human granulocyte colony stimulating factor, may influence practice in Cape Town. At the same time it was motivating to exchange ideas on our currently active programme for harvesting haematopoietic stem and progenitor cells from the peripheral blood. Here again, the anticipation by Professor Jacobs of introducing continuous blood flow technolog more than a decade ago and establishing entire cryopreservation programme has made it a relatively easy step for us to convert research and development in these areas to clinical treatment programmes.

\section{THE PROTECTED ENVIRONMENT FOR MANAGING IMMUNO- COMPROMISED PATIENTS}

Information has been pooled from the Daniel Den Hoed Cancer Centre, Rotterdam, Holland, the University Hospital in Munster, Federal Republic of Germany, the Kanton Hospital in Basle, Switzerland, supplemented by discussion at the congresses attended.

\section{Systemic Antibiotic Programmes}

In these specialised facilities the locally occurring microbial flora are monitored as the basis for individual programmes, as is the practice in Cape Town. Such an approach implies regular involvement of microbiologists as part of the multidisciplinary health care team. Patients developing a significant fever are treated with an 
aminoglycoside and a second or third generation cephalosporin combination. Samples are taken on a regular basis, usually from the central venous catheter, but on occasion from peripheral lines as well. There is strong support for routine surveillance blood cultures, examination of oral mouth washings, vaginal swabs and microbiologic study of urine and faeces at least weekly. In general terms, there is increasing domination of the isolates by gram positive over gram negative organisms; Staphylococcus epidermidis is a particularly troublesome pathogen in all centres.

Another favoured approach is to commence empiric antibiotic regimens automatically when granulocyte counts reach $0,5 \times 10^{9} / \mathrm{L}$. Concurrently, patients received microflora, which is a special preparation designed to reintroduce selected bacteria into the sterilised gastrointestinal tract.

Monotherapy using Imepenem, as opposed to antibiotic combinations for the neutropenic patients with pyrexia, has not been clarified. However, in extensive discussion with microbiologists it seemed that the programme c ntly used in Cape Town is appropriate anturt is notable that our practice is constantly under review by the Infectious Disease Service.

\section{Antifungal Agents}

The use of these drugs was constantly debated. Many of the group use amphotericin B prophylactically from the time that the total white cell count reaches $0,5 \times 10^{4} / \mathrm{L}$. Although nephrotoxic, there are new formulations that include its encapsulation in liposomes or administration in combination with intralipid. Little information on any of these products seems to be readily aváilable in Cape Town and our group are exploring the possibility of obtaining one of these or a recently released and apparently less nephrotoxic analogue. It may well be realistic for us to compare the various formulations of amphotericin in a trial.

\section{Antiviral Prophylaxis}

It was re-emphasized that, particularly following transplantation, the immunocompromised and neutropenic patient is increasingly at risk, particularly when additional management problems are created by acute or chronic graft-versus-host disease. Since some of the hazards relate to cytomegalovirus reactivation or the introduction of new infection, screening of blood products is important. Furthermore, the use of intravenous gammaglobulin before and after transplantation is a sensible way to protect the patients from this hazard. Some concern was caused by the report that DHPG did not decrease the mortality when cytomegalovirus occurred, although it appears to diminish the frequency with which the complication arose. A point of practical importance was how best to combine this myelotoxic agent with the gammaglobulin. It is clear that material must be obtained for study, either by bronchial lavage or, wherever there is uncertainty, by recourse to bronchoscopy with biopsy or to obtain lung at thoracotomy.

\section{Selective Decontamination of the}

\section{Gastrointestinal Tract}

This is considered in every way preferable to attempts to absolutely sterilize the lumen of the bowel. This practice is applied to all neutropenic and immunocompromised patients, whether they are undergoing chemotherapy for haematologic malignancy or following bone marrow transplantation. In general, this programme commences prior to chemotherapy or conditioning with drugs or radiotherapy and aims to specifically maintain colonisation with anaerobes at a level of $1 \mathrm{x}$ $10^{6} / \mathrm{mL}$. Combinations favour nystatin, and either tablets or suspension can be used every 6 hours at a dose of $2 \times 10^{\circ}$ or

$1 \times 10^{5}$ units, respectively; these programmes continue until the patient is in complete remission or for 6 months after grafting. A useful alternative is amphotericin $B$, at a dose of $500 \mathrm{mg}$ every 6 hours with tables or 100 $\mathrm{mg}$ if the suspension is used. A third alternative is polymixen every 6 hours, with $400 \mathrm{mg}$ norfloxicin twice daily. Wherever possible, all agents are prepared in suspensions and administered using the swill and swallow technique, trying to keep the product in the mouth for 15 minutes, although this is often fairly difficult.

Complete decontamination is reserved for patients undergoing transplantation using mismatched donors and where absolute sterility is maintained in plastic isolators. Here the attempt is to remove all bacterial or other colonies, but this is a procedure having its own risks. Specifically, should patients develop an infection, they are less well able to deal with it. A currently favoured regimen is to give $500 \mathrm{mg}$ of neomycin together with $500 \mathrm{mg}$ of keflin and $2 \times 10^{\circ}$ units of nystatin every 6 hours.

\section{The Dedicated Dietician}

All groups acknowledge that the proper operation of a protected environment requires participation from many paramedical professionsals. These include social workers, psychiatry-liaison consultants and occupational therapists. Additionally it is necessary to have a full time or at least dedicated dietician, who will be working in association with a food services manager. I was interested to see these recommendations, since this was one of our early priorities.

In Cape Town we are currently seeking to achieve three specific objectives.

Firstly, to ensure that the food is palatable and that it is delivered to the unit in a way that makes warming in a conventional or microwave oven possible. There are substantial difficulties with achieving this goal and we in Cape Town are seeking to overcome some of these. This is important since the patients are ill, have disturbances in taste physiology, and varying degrees of anorexia and nausea are present. Studies in factories were reported on how food should be delivered, temperatures required for sterilisation during preparation, and the listing of safe products. Integral to this part of our programme is the need to ensure that patients are instructed and understand the practice since, at least in bone marrow transplanted individuals, this would need to be continued after their return home and whilst on nystatin for at least 6 months.

Secondly, there is a hazard to contaminating the bacteria-free diet by carelessness that might include the supply of salads, which are recognised carriers of pseudomonas, fresh fruit from which the peel and core has not been removed, or food brought into the unit by well-intentioned family members. Since the latter procedure is often secretive, patients are placed at risk, and education of the individual as well as visitors and nursing staff by the dietician is an important consideration.

Thirdly, dieticians play a central role in enteral as well as total parenteral nutrition. Essential contributions are to establish and maintain basic caloric intake both before and throughout the procedures, and here daily weights are important. Other measurements of nutritional status require measurement of serum protein, pre-albumin and albumin, bearing in mind that the latter fluctuates because of stress, during pyrexia and following blood product infusion. A priority is the early commencement of patients on whatever dietary support programme is necessary to contribute between 1600 and 2000 calories daily and to increase this as patients switch to oral feeding, which should occur as soon as possible. Tube feeding is not widely favoured because of the discomfort, and whenever anorexia, nausea, vomiting or diarrhoea develop this is in any event replaced by the parenteral route. All crockery and cutlery needs to be carefully sterilised.

\section{Central Venous Lines}

There is no question in the mind of any group operating the kind of programme that we run in Cape Town, including protected environment, that double lumen central venous catheters are an absolute prerequisite for optimum care of these individuals.

However, central venous lines are not without problems and as we and others are aware one area of particular difficulty is the increasing incidence with which Staphyloccocus epidermidis is isolated. The early enthusiasm for removing lines has been largely replaced by a more cautious attitude, aimed at the use of appropriate antibiotics. This practice has its basis in the general observation that once bone marrow recovery occurs with granulocyte production, the patients can clear this pathogen with little difficulty. With the local unit in the process of being commissioned, very particular attention will now be given to the placement of lines under the sterile conditions that prevail in the operating theatre and that all subsequent manipulation be carried out in a similar manner.

Another issue that I was able to discuss is the concern expressed in our department about the use of vancomycin. It is notable that our own practice is, if anything, more highly controlled than many units worldwide where this agent is used prophylactically in the neutropenic patient who develops a fever even though blood cultures are negative. There was no reason evident from any of the discussions I had specifically on this point or from presentations that the practice we have established for the placement and care of venous lines and the use of vancomycin is 
anything other than consistent with widely endorsed policies - if anything, even a little conservative.

\section{General Considerations}

Many of the points discussed at the meetings and summarised above have relevance for the continuing commissioning of the protected environment in Groote Schuur Hospital. Thus, traffic in the overall unit must be carefully controlled and visiting needs to be meticulously supervised.

It is mandatory that the staff are not only highly motivated but have access to continuing in-service training which should include interaction with social workers and psychiatrists. The latter should take into account not only patients problems, but the stress that may arise amongst doctors and nurses working for protracted periods in protected environments with very ill patients. It is, however, very important that these groups in no way disturb the overall responsibility that resides with the head of the department. The recognition of any interpersonal relationships or disturbed interaction between medical and nursing colleagues should be resolved departmentally, rather than allowing the emergence of splinter groups that might actually turn out to be counterproductive. Attention to these areas is proving useful in our own department.

Similarly, antimicrobial programmes are under constant review and both dietician and food services manager should make regular clinical ward rounds as an integral part of developing a cost-effective approach to selective decontamination of the gastrointestinal tract.

Two other issues are currently under critical review. The first of these is the placement and care of our central venous lines. Here, it is notable that in some centres vancomycin is used relatively early since it is emerging with increasing clarity that this will diminish gram-positive infections, particularly with Staphylococcus epidermidis. Secondly, it is equally clear that if we are to reduce colonisation with fungi, then selective decontamination of the bowel is necessary, and we may well need to use parenteral antifungal therapy prophylactically.

One final comment is appropriate. Having compared single rooms to laminar-flow facilities and isolators it appears to me that the design of our unit in Cape Town is in every way comparable to the best that I visited or heard discussed. The most immediate challenge now facing us is to be certain that the operation of the protected environment is optimal in order that we can realise its full potential.

\section{CONTINUOUS - FLOW SEPARATOR TECHNOLOGY}

These programmes are integral to all haematology departments having made a commitment to the management of patients with haematologic malignancy and that now fairly routinely employ bone marrow transplantation. In addition, the availability of this technology makes it possible for a number of acute and often fulminating antibody-mediated diseases to be treated by plasma exchange. In view of the extensive and ongoing commitment that we have in this area it was relevant to compare our practices to those established in other centres.

\section{Instruments and Staff}

There is every reason to believe that the selection of the IBM and more recently the Cobe series of instruments has been a correct one. With the development of the new generation separators in the form of the Spectra we can confidently anticipate maintaining the reputation that the department has developed since introducing the technology into the Republic more than 10 years ago, and subsequently to running educational symposia and providing training for others throughout the country.

One of the other instruments discussed was the Hemonetics V5O, which is fully automated. The shortcoming, in my opinion, was the fact that at any one moment $500 \mathrm{~mL}$ is outside the system and on reinfusion of plasma the patient may experience toxic effects from citrate anticoagulant. It was of interest that these symptoms are managed not by calcium replacement but by reducing the return flow from the machine. Red cell contamination is held to a minimum and platelet yields range between 3 and $4 \times 10^{11} / \mathrm{L}$.

On reviewing the Fenwall CS3000 I did not form the opinion that there were any advantages in switching machines in the foreseeable future.

In regard to staff, whether this be training or operation of the instruments, I remain convinced that this is best done by nurses as opposed to technicians or technologists. This opinion is based on the fact that where difficulties with resuscitation occur and bearing in mind that many of the patients are seriously ill, there is no substitute for an experienced nursing team. The ongoing programme that we have in our department is, in my judgement, consistent with optimal practice when compared to other specialised units visited or based on discussions at the international nurses' meetings.

\section{Platelet Transfusions}

These are given prophylactically in some centres at levels of $20 \times 10^{4} / \mathrm{L}$ and the 1 and 24-hour post-infusion increments documented. We have shown in prospective controlled studies that this is one of the most important ways for maintaining quality control and it is essential to combine these observations with a knowledge of the number of platelets infused.

In an attempt to reduce isoimmunisation, single donor platelets are used and gamma irradiation is necessary to diminish the risk of developing graft-versus-host disease. This facility exists in our group at Cape Town, where a gamma-cell cobalt source delivers 15 Gy prior to infusion.

An interesting trial, similar to that which is now being developed in our department, is to prospectively study the responses of patients who become sensitised, and here pretransfusion testing of the donor is carried out using an enzyme-linked immuno-assay or, in isoimmunised patients, the use of partially matched platelets. The current technique is not yet perfect and a number of groups are trying to develop better assays. Of note is the fact that both family members and a donor panel are maintained from community volunteers and we have found this to be practical in Groote Schuur Hospital. To safeguard those individuals, twice weekly donations can be made provided the precollection level is known.

One interesting innovation was the use of filtered platelets to reduce leucocyte contamination in those who have not had prior cytomegalovirus exposure. The rationale for this is to diminish the direct transport of the virus into the patient due to contamination with granulocytes.

\section{Graft-versus-Host Disease (GvHD) \\ Reduction}

Attempts to avert this often lethal complication of transplantation or even the infusion of immunologically competent cells into an immunosuppressed recipient is managed in most centres by irradiation of blood or blood products. An interesting study has recently been reported where it appears possible to achieve an equal degree of protection by exposing the units to ultra violet light before transfusion. Our group have been involved in discussions with investigators at Georgetown University, and this remains a potentially fruitful area for future collaborative study.

\section{Buffy Coat}

In some of the centres peripheral blood cells are collected and administered to bone marrow transplant recipients following T-cell depletion. Most of these programmes require irradiation of the product to circumvent the development of severe acute graft-versus-host disease. There is some evidence, preliminary at the present time, that addition of this manoeuvre favours more rapid regeneration of peripheral blood values. Buffy coat is administered every second or third day and the number of cells gradually escalated; it is currently not clear what the rationale for this procedure is since irradiation would effectively prevent stem cell regeneration, uid it therefore remains likely that it reflects the in vivo maturation of progenitors already committed to granulocyte differentiaton.

\section{ABO Incompatibility}

In earlier years differences in red cell groups between donors and recipients were overcome by continuous-flow exchange against group AB plasma. Other options were selective removal using immunoadsorption columns, whereas recent studies have been initiated to refine the techniques by in vivo absorption with titrated doses of incompatible red cells. It seems to me that this is a hazardous procedure, even though the cells are given slowly, particularly since patients develop fever and back pain. Nevertheless, titres can be markedly reduced.

In Cape Town we have now accumulated some experience in the role of continuousflow plasma exchange for reducing naturally occurring antibodies in donor and recipient pairs where $\mathrm{ABO}$ incompatibility would otherwise be an insurmountable problem. It has been shown that both under these 
circumstances and where significant titres of lymphocytotoxic antibodies are present the Cobe 2997 is a highly efficient machine for achieving reduction of antibodies to a level that renders allogeneic transplantation safe.

Technically we have found two consecutive exchanges are adequate, although some groups simply ignore the presence of antibodies where T-lymphocyte depletion grafts are used on the basis that red cell contamination is inconsequential. The latter approach is satisfactory in that no haemolysis would occur until such time as engraftment is established. However, the new red cells are susceptible to immune-mediated destruction from any residual circulating antibodies. We believe that it is best to en on the side of conservatism, although our experience is consistent with that reported elsewhere in which titres of less than 1 in 16 can generally be ignored.

\section{Allogeneic Granulocyte Infusion}

This product is not used a great deal since many investigators feel that currently Yable antibiotics are sufficient and, d, some of the complications may outweigh the benefits. An example of the latter is the administration of hydroxyethyl starch and corticosteroids to the donor, making it impractical to re-utilise these individuals more than four times a year. Of note is our experience, which is clearly in contrast to this, since we believe that in carefully selected patients allogeneic granulocytes may be effective and indeed lifesaving. The group at Groote Schuur Hospital have, however, never carried out a large prospective randomised trial to exactly define the use of this practice, mainly because the logistics would be formidable.

\section{Plasma Exchange}

This technique is widely used, particularly for the control of hyperviscosity in patients with myeloma, where a prerequisite is measurement of plasma or whole blood value. F rably, together with the development of symptoms. Reports show that patients require these procedures weekly and at least one group of investigators favours the Fenwall CS3000, which is fully automated. I, however, found it cumbersome and particularly noisy. It was interesting to examine our own substantial experience and we believe a much more scientific approach has been developed at Groote Schuur. We are currently extending our studies, using plasma exchange standardised to body surface area and also examining the role of sham procedures. This study is being planned collaboratively with Professor J P Crowley at Browne University and will include psychometric evaluation as well as assessment of machine efficiency and changes in whole blood viscosity.

These procedures are performed in some areas for Guillain Barre syndrome and I was unsure as to how some of the trials were being conducted. For example, the referring physicians operate independently and give the patients immunosuppressive drugs without directly involving the cell support staff and, as a consequence, the relative contributions of the two forms of therapies cannot be established. Our own practice has been to rigidly control the patients in terms of immunosuppressive therapy and replacement technique; studies in Cape Town are invariably conducted on a collaborative basis with the respiratory group and neurologists.

\section{Disseminated Intravascular Coagulation} This is a problem that frequently involves infected patients and I did not encounter any practices that were significantly different from our own. However, as was evident in all the centres visited, the nursing staff have a major role to play in meticulously managing fluid balance, avoiding sodium overload and carefully charting the replacement of various clotting factors. Up to this point, international practice was similar to ours, but we are planning to examine, under controlled circumstances, the theoretical benefits of plasma exchange in patients with acute leukaemia. particularly the progranulocytic variant, where release of large amounts of thromboplastin into the circulation is particularly hazardous during induction chemotherapy.

\section{Lymphokine Activated Killer (LAK) Cells}

A number of programmes exploring biological immune response modulation rest upon continuous-flow separator technology. In this particular programme lymphocytes are harvested from the peripheral blood and following stimulation by growth factors, such as interleukin 2, the activated natural killer cells fraction can be recovered. This is an area that our group will be discussing with Professor Jeane Porter Hester when she visits early in 1990, since there is preliminary evidence that infusion of these cells may be effective, not only in patients with haematologic malignancy but in a number of those with solid tumours. This approach well illustrates the increasing service that haematologists currently provide for colleagues in other disciplines - in this example, the solid tumour oncologists.

\section{Procurement of Circulating Haemato- poietic Stem and Progenitor Cells}

As elsewhere in the world, one of the most rapidly developing areas utilising continuous blood-flow technology is for the recovery of a population in the peripheral blood suitable for bone marrow transplantation. The success of this procedure would mean that harvesting cells from the bone marrow under general anaesthetic might, in the future, no longer be necessary.

Our own phase I studies in this regard are completed, and it was therefore helpful to be able to critically examine results from other investigators. We have found it unnecessary to stimulate the marrow in our donors with granulocyte colony-stimulating factors, although in some centres this is routine. It may well be that in patients with myeloma this particular practice is important, but we have not, to date, examined recovery in patients with this haematologic malignancy.

In the lymphomas, patients aged between 15 and 60 years have been entered on trials and cells collected after two course of a variety of regimens, many of which contain vincristine, adriamycin and dexamethasone. Prior to stem cell harvesting in this group, as with patients having myeloma, the priming with growth factors has been found helpful. One of the protocols is a collection procedure carried out for six consecutive days, aiming to harvest in the region of $2 \times 10^{\circ} \mathrm{GM}: \mathrm{CFUc} / \mathrm{kg}$. This product is then cryopreserved, using dimethyl sulfoxide.

A variation that I observed with interest was the collection of circulating mononuclear cells, which is the fraction that incorporates the haematopoietic stem cells, using continuous-flow separation, with the erythrocytes simply being returned to the donor. This is our current practice, but some of the investigators had an additional step seeking to enrich the population using density-gradient separation techniques. We do not believe this has any role and raises some problems about potential contamination.

Our phase II study is currently in progress documenting the exact schedule for optimum collection procedures in individual patients and defining any damage that may accrue from the use of dimethyl sulfoxide as a cryoprotectant. Further studies are also to confirm the adequacy of the yield for autografting.

In the final projected phase III we will prospectively and randomly evaluate the role of folinic acid and also the need to combine infusions of stem cells harvested from the peripheral blood with that of marrow.

\section{BONE MARROW TRANSPLANTATION}

\section{General Comments}

Allogeneic or autologous grafting are well established and, indeed, currently the form of treatment dominating management of haematologic malignancy. It is noteworthy that a similar approach is permeating the solid tumour oncology field, largely as a means of providing haematopoietic reconstitution following escalation of radiotherapy and cytotoxic drug administration to levels never previously possible because of their creating irreversible bone marrow damage.

The success of these programmes is contingent upon the availability of a number of inseparable components. Firstly is a cell support section incorporating dedicated facilities for irradiation of the collected products, with the latter necessary to reduce the incidence and severity of graft-versus-host disease. Secondly, a proper facility in the form of a protected environment is important, with the provision of laminar air-flow rooms or isolators as a major contribution to improving survival of patients from the neutropenic period. Thirdly, and usually considered as part of the protected environment, is the protocol management of infecions that incorporates placement and care of central venous lines, prophylactic and therapeutic antimicrobial drug administration and enteral and total parenteral nutrition as well as food management. Fourthly is the need to have a dedicated multidisciplinary health care team to maintain high standards of continuing medical education and ruthless supervision, monitoring and implementation of traffic control and behavioural practice within the unit. It is in this latter area that 
breakdown most commonly occurs, due to poor quality janitorial services and irresponsible behaviour by nursing and medical staff, both of which areas necessitate constant attention to detail. Fifthly, the presence of paramedical colleagues on a regular basis as part of the patient care group involves social worker, physiotherapist, psychiatry liaison, food services manager and constant interaction with other disciplines such as renal and chest physicians. Sixthly is the availability in the unit of whatever intensive care support is necessary since the major clinical problem is that of an immunocompromised patient and such individuals should not leave the unit. Furthermore, because of pressure on other facilities, it is more practical to have the primary responsibility rest with the haematologists and then to consult with whatever other disciplines may be required.

It is a credit to our university and hospital that the vision to plan this unit, extending back to more than the 10 years that I have been in the department, is now a reality. None of the centres I visited had major differences from our practice, but in each there was strong endorsement for the principles set out above. It is now a matter of our department working diligently and ceaselessly to maintain all the standards necessary for this unit to operate in a cost-effective manner.

\section{Haematopoietic Growth Factors}

The use of granulocyte or granulocyte: macrophage colony stimulating factors, among many others, has given rise to a whole new area of haematologic research and development. These products, which are available in recombinant human form for the large part, have the ability to increase the white cell count, but raise a number of interesting questions. It remains to be clarified as to whether concurrent and unwanted stimulation of the leukaemic clone may occur at the same time as normal regeneration in patients receiving these for haematologic ma lignancy. In the myelodysplastic syndromes these biological reagents are particularly interesting, and again the question of not only producing induction of maturation from the tumour but also leading to its acceleration has not been completely answered. The latter effect appears to be dose related, and in the studies currently in progress in our department this point is being closely observed. There is some evidence that combination of these agents with low-dose cytosine arabinoside may be a particularly hazardous practice, but even this observation is currently without confirmation. It has been suggested that while the overall time to reach 0,5 or $1 \times 10^{7} / \mathrm{L}$ granulocytes is shortened, the critical period of reaching $0,1 \times 10^{4} / \mathrm{L}$ may not be changed. Since it is this latter interval that is associated with most mortality, more information on scheduling is necessary. It is also not clear as to whether the increased number of granulocytes that arise from such stimulation are functionally normal; this is also the subject of study in our own laboratory. It was a bit disappointing to find that when used in patients with aplastic anaemia it has little benefit; most bringing about an increase in eosinophils, with very little effect on other lines.

Even with these concerns the steady march of haematology into the field of cellular and molecular biology was striking in all the presentations at the meetings and occupied most of the discussions around the management of patients with haematologic malignancy or following bone marrow transplantation. It was clear to me that, as with all the areas in our department, the direction in which our activities are being guided is in keeping with the best international standards as I gauged them on this trip. This observation is also consistent with the viewpoint expressed by the steady stream of visitors who come to the department, bringing with them, from all parts of the world, expertise in practically every aspect of the work going on in Cape Town.

\section{Patient and Staff Education}

The successful management of patients on a transplantation programme has two other important considerations.

It is essential that all members of the multidisciplinary health care team are confident that the programme offers the best choice for the individual patients. To this end there need to be regular discussions in the department and particularly morbidity and mortality rounds should explore any problems to clarify the circumstances of death and, as far as possible, prevent recurrence. This has been one of our long established practices and I was rather surprised to find that in most other centres similar attention is not given on a regular basis to the outcome of treatment, although ongoing results are reviewed.

Not infrequently the patients entering these programmes do so without being adequately preptred. Our own practice is to have everybody interviewed by our social worker and liaison psychiatrist so that we can anticipate disruption in the family structure and cater for it appropriately. Furthermore, I was again a little surprised to see that virtually no attention is given to detailed analysis of results and their presentation at meetings although, as far as I could gauge, carefully informed consent is provided in many centres. One of the areas that needs further attention in our group is the information and care of the bone marrow transplant donor. These individuals are often, as I judge it, insufficiently advised of their vital role in management. This issue is now to receive further attention in our department.

Another area where we have a particular interest but which is not well covered in presentations, although it appears to be under consideration by different groups, is that of confidentiality. We have become aware that this matter is inadequately appreciated now that the protected environment is open. Already major efforts have been made to improve what we all recognise as an area of considerable sensitivity and where continuing medical education is going to have to play a much bigger role.

\section{Conditioning Regimens}

While these vary widely from centre to centre and between disease categories, some general comments are possible. The use of total body irradiation in combination with cyclophosphamide retains pride of place. Nevertheless, there are important alternative options currently being explored where myleran, high-dose cytosine arabinoside or even anthracycline antibiotics are considered. The emphasis in conditioning regimens is twofold. On the one hand is the need to produce the required degree of immunosuppression so that the infused graft will not be rejected. On the other, applicable only to patients with haematologic malignancy, is the need to eradicate all residual disease. Since patients with acute leukaemia undergoing transplantation have a significant disease recurrence, this consideration is being actively investigated. It was disappointing to find that modifications to the well-established total body irradiation and cyclophosphamide regimen have little to offer. However, of particular interest is work from the Johns Hopkins group showing that the use of myleran in combination with cyclophosphamide seems to have a lower incidence of this complication, but toxicity is significant, particularly in previously untreated patients.

Of great interest to us because of more than 15 years' experience with the epipodophyllotoxin VP16-213 are regimens that combine total body irradiation with a single infusion of etoposide given three days before the graft, with dosages in the region of 50 to $70 \mathrm{mg} / \mathrm{kg}$ and usually following total body irradiation. Whilst mucositis was a problem in $96 \%$ of patients and diarrhoea in $50 \%$, together with some degree of hepatotoxicity, this regimen seems to have some additional advantage as an anti-leukaemic agent because of its ability to cross the blood-brain barrier. Its value in acute nonlymphoblastic leukaemia remains controversial, but appears less satisfactory

When all of this material was reviewed against the experiences in our own group I was again gratified to find that local practices are consistent with those considered optimum by all other investigators. We will have to continue with a disciplined approach in orde that sufficient data can accrue on our prot for analysis and modification once statistical significance has been achieved. It was striking to see how poorly organised many of the studies were and the willingness with which investigators changed without any real basis for such variations. This practice contrasts with the really large centres, where the protocols are rigidly implemented and, as a consequence, authoritative statements can be made about experience, at least in that particular centre. It is frustrating to find, as we have known for many years, that where apparently comparable regimens are used in two or three centres, side effects and outcome vary widely, so that we remain dependent upon data from the International and European Bone Marrow Transplantation Registries for providing much of our developmental work. The active involvement of our centre, particularly in the IBMTR, reflects creditably upon our university and hospital and has the additional advantage of making available to us on a regular basis preprints of data that to an increasing extent involve our own patients. 
Acute Nonlymphoblastic Leukaemia (ANLL) and Myelodysplasia

Controversy persists as to the relative merits of modern aggressive chemotherapy regimens compared to bone marrow transplantation.

There seems to be a consensus of opinion that if grafting is to be used, this should take place as early as possible after the patient is in consolidated complete remission. After the first six months the differences are diluted, probably because after this period in continuous remission a subgroup of patients has been biologically selected who will not benefit from grafting. However, by waiting for this time to elapse, a very large number would be at risk from leukaemic relapse. Our own practice is, therefore, to proceed to graft wherever possible and to do so as soon as possible; this would reflect the approach of most of the major centres worldwide.

As with de novo ANLL, patients with certain subgroups of myelodysplasia benefit significantly from allografting. A much more difficult challenge is exploration of autologous or double-autografting in these patients; both approaches are currently Jergoing evaluation in our department. It is necessary to pay further attention to the quality of life after transplantation, and at least one centre is generating a questionnaire to be completed at the 6 months' follow-up visit, that will examine performance states and sexual function. In anticipation of damage to germ cells we, and many others, advise our patients of the hazard and in males arrange sperm banking.

Another area which is being examined is ex vivo graft manipulation. Some programmes report high relapse rates and therefore expose the stem and progenitor cell to

cyclophosphamide or one of its derivatives, whereas other groups are following an essentially similar approach but using monoclonal antibodies. Our own practice, using the Campath $1 \mathrm{M}$ reagent developed by the molecular biology group at Cambridge. with whom we continue to collaborate, remains an acceptable programme and we are unaware of any reason to change it at the ent time.

The introduction of autologous transplantation is steadily emerging as being of major clinical value. The repeat or double autografting procedure, which we plan to use, is established when patients enter on study. Information from other centres demonstrates that not all individuals reach the second graft, but where survival curves are available this is comparable or even superior to allogeneic bone marrow transplantation, irrespective of the procedure used. The theoretical basis for the better results may lie in greater disease reduction from two widely different conditioning regimens. Alternatively, there is increasing evidence that autologous grafting favours the early return or even increased incidence of killer and natural killer cells selectively, which differs from allogeneic grafting with or without unfractionated bone marrow, where this cell population returns slowly towards normal. Since this is the cellular mechanism currently thought to significantly influence leukaemic relapse, our practice of double-autologous grafting would appear to have additional the oretical support.

In the context of allografting, all centres are intensively concentrating on harvesting of stem and progenitor cells from the peripheral blood. In our own group the experimental basis for this practice has been firmly established and studies are in progress to transfer the laboratory experience to the clinic. Amongst the unsolved areas is the possible role for pretreatment of the patient with growth factors, but it remains to be determined what advantages this procedure will have.

Of particular interest to ourselves and other groups is the unanswered question as to whether repopulating cells are derived from marrow aspiration. The most attractive data available suggest that the two populations are different and therefore their use in combination may significantly shorten post-infusion neutropenic periods. This practice is to be explored in a prospective controlled randomised study in our group.

\section{Acute Lymphoblastic Leukaemia}

Very little difference exists at other centres from our established practice in Cape Town. Thus, the recognition of high risk groups and their early allogeneic or double autologous transplantation is, for the moment at least, consistent with the best treatment that can be offered to these patients.

\section{Chronic Granulocytic Leukaemia}

The management of this disease has undergone dramatic change in recent years with the demonstration that allogeneic bone marrow transplantation offers, for the first time, a chance for cure. This is an area of particular interest since unfractionated marrow is associated with an unacceptably high and often devastating incidence of acute graft-versus-host disease. Attempts to offset this lethal complication by $e x$ vivo graft manipulation have given rise to heated controversy. There are claims that leukaemic relapse is a problem and counter arguments that this is a function of the purging technique used.

Like the Dutch group, we have chosen the altemative route of attempting to reduce residual leukaemia by three courses of combination chemotherapy prior to transplantation. Our own studies, while consistent with the approach taken by two other major centres, are too early for interpretation. However, local efforts are directed at trying to diminish the malignant clone as reflected in karyotypes expressing the Philadelphia chromosome. There is some evidence from other investigators that the development of a mosaic between normal and abnormal cells on cytogenetic studies is associated with a lower relapse rate following transplantation.

A further point of interest and currently unexplained is the observation that following grafting a number of patients revert to the chronic stable phase of the disease, but such cytogenetic relapse does appear to progress to clonal expansion. It has also been observed that some patients relapse with acute nonlymphoblastic leukaemia, particularly where they have previously received recombinant human alpha-interferon in an attempt to reduce the leukaemic clones.
It is clear to me that we need to enter 20 or 25 patients on our own programme and analyse that data before we arbitrarily change because of something that one or other group is reporting but which is without confirmation.

\section{Chronic Lymphocytic Leukaemia}

This remains a subject of intensive investigation and groups are using bone marrow transplantation with T-cell depletion with apparently successful outcome in younger patients. This is interesting, particularly where autologous or double grafting procedures are employed. Ex vivo manipulation with monoclonal antibodies is being explored in some centres, but no consensus is available and management, for the moment at least, needs to be on the basis of local protocols.

\section{Hodgkin's Disease and Non-Hodgkin's Lymphomas}

As referred to in the earlier section where disease categories were discussed, it is clear that bone marrow transplantation is to be increasingly employed in these patients. In recent years it has become clear that subgroups of patients, including those who respond slowly in both categories of malignant lyniphoma, have a high relapse rate with all available chemotherapy, whereas a percentage may be cured with bone marrow transplantation.

In the planning of the protected environment the needs of the joint myeloma and lymphoma clinic were specifically catered for and in our university and hospital it is now a reality to offer this service for suitably selected patient.

\section{Non-identical Donors}

Two points are of relevance. In the first, related but HLA non-identical sibling donors are used. However, as the antigenitic disparity rises so does graft failure, which may reach $60 \%$. Furthermore, there is a marked increase in the incidence of acute graft-versus-host disease as the number of disparate antigens between the transplant pairs widens.

The second alternative is to use a phenotypically identical but genotypically different or unrelated donor. Registries are being developed to make available information about such individuals; the major problem remains graft-versus-host disease. Unrelated donors have a high incidence of this serious complication and their survival is also less good, particularly for aplastic anaemia. In contrast, related donors with varying degrees of antigenic disparity have $30 \%$ and $10 \%$ respectively of grade I and II or grade III and IV GvHD, but with a $100 \%$ oneyear survival.

\section{Acute Graft-versus-Host Disease}

This remains an enormous problem, with attempts to abrogate its incidence and severity with antilymphocyte globulin, prednisone and a variety of monoclonal antibodies, all relatively ineffective. More recently, attempts to block the interleukin 2 receptor with a monoclonal antibody have been encouraging 
and results of a randomised trial are awaited with interest.

\section{Chronic Graft-versus-Host Disease}

Treatment of chronic graft-versus-host disease remains problematical, with no advances on management with prednisone, with or without azathioprin; there is an increased risk of infection and consequently reduced survival. Prednisone and cyclosporin are alternative treatments, but have met with limited success. Immunoglobulin prophylactically seems the most promising, and a potential role for thalidomide is being explored, but data are presently limited. The problem is that the latter agent is difficult to obtain and side effects range from mild neuropathy and drowsiness to severe headaches and coma; these can be reversed on withdrawal of the drug. Response rates are claimed to be as high as $70 \%$, particularly in the skin, and it may have a role for steroid sparing and is non-myelosuppressive.

\section{Patient Briefing in Bone Marrow Transplantation}

Most departments are currently making a great deal of effort to provide a multidisciplinary approach and to discuss in detail with recipient, donor and family all aspects of the procedure.

\section{Visit to University Hospital in Bern}

I had an opportunity to discuss with Dr Paul Imbach our previous ITP studies and to review a new prospective randomised trial that we are about to initiate. There was much interest as to the exact role that high-dose immunoglobulin might have in treating adult patients with chronic idiopathic immune thrombocytopenia. Although there is consensus that these patients should initially be treated with corticosteroids, uncertainty exists as to the long term benefits that may be determined by the sequence in which individuals receive immune modulation with immunoglobulin or undergo splenectomy.
Whilst visiting the Central Laboratory of the Swiss Red Cross Hospital it was interesting to see how the immunoglobulin that we use is manufactured. Blood is collected from donors all over Switzerland by mobile vans and once processed this product is widely distributed throughout the world. It is of note, and some concern, that HIV is prominent in Switzerland, but all the donors are meticulously screened and the techniques for preparation of the product are directed at viral inactivation. The only paid donors in this programme are those who contribute anti-D, whereas anti-tetanus hyperimmune globulin is obtained from army volunteers.

Throughout Switzerland there are regional services in hospital which supply their own platelets, but few institutions have as well organised cell support section as operates at the University of Cape Town and Groote Schuur Hospital.

\section{The Role of the Professional Nurse} Naturally I was particularly interested to see the career structure that exists for senior nursing faculty. It was striking that while much more efficient use is made of part-time professionals and employment on flexitime, the academic aspect of our profession is less well developed than in the United States of America.

\section{SUMMARY AND CONCLUSIONS}

Haematology is one of the most rapidly expanding disciplines in medicine and nursing. As occurs in other highly specialised areas, optimum care is now largely of a multidisciplinary nature. In this context there are literally unlimited opportunities for the involvement of professional nurses and, as I have attempted to illustrate in this report, integration in all aspects of research and development and active participation in presentation of research data and discussion at international meetings is one direction in which fulfillment of academic aspirations can be achieved. It is my viewpoint, based on more than a decade of direct involvement in all the activities of our department in Cape
Town that these are entirely attainable goals. There is currently, in our country, a concerted move afoot to develop an improved career structure for the professional nurse along the lines of the American clinical nurse specialist. Much of this experience overseas would strongly support that commitment.

It was my privilege to enjoy the confidence of the department, university and medical school sufficient for me to present research data at international meetings and to be a welcome visitor at some of the world's premier academic and research institutions. That this was possible reflects the uncomprising commitment in Haematology to the position of the professional nurse as an integral and equal part of the multidisciplinary health care team.

\section{ACKNOWLEDGEMENTS}

I express appreciation to Professor Peter Jacobs for encouragement and confidence in making it possible for me to present departmental research at the Bone Marrow Transplantation Meetings and I am grateful for the opportunity that was afforded me by Groote Schuur Hospital for leave privileges and financial assistance. My thanks go to Miss June du Preez, Deputy Director Nursing, for her support, the Nurses Fund for payment of registration fees at the two international congresses, the University of Cape Town for additional travel and subsistence and to Jackie Davies for help with preparation of the report and its typing.

Lucille Wood, R.N., R.M.,

Chief Professional Nurse from The University of Cape Town Leukaemia Centre and the Department of Haemarology, Groote Schuur Hospiral.

Supported by the University of Cape Town Leukaemia Centre and Staff Research Fund, the Gwendoline Moore Trust, the Medical Research Council, the National Cancer Association and Michael Chanani and Kaliski Bequests 